\title{
BMJ Open Development of the CLARIFY (CheckList stAndardising the Reporting of Interventions For Yoga) guidelines: a Delphi study
}

\author{
Lesley Ward,,$^{1,2}$ Daryl Nault, ${ }^{3}$ Holger Cramer (1) , ${ }^{4}$ Steffany Moonaz (D) ${ }^{3}$
}

To cite: Ward L, Nault D, Cramer $\mathrm{H}$, et al. Development of the CLARIFY (CheckList stAndardising the Reporting of Interventions For Yoga) guidelines: a Delphi study. BMJ Open 2022;12:e054585. doi:10.1136/ bmjopen-2021-054585

- Prepublication history and additional supplemental material for this paper are available online. To view these files, please visit the journal online (http://dx.doi.org/10.1136/ bmjopen-2021-054585).

Received 17 June 2021 Accepted 22 December 2021

Check for updates

(C) Author(s) (or their employer(s)) 2022. Re-use permitted under CC BY-NC. No commercial re-use. See rights and permissions. Published by BMJ.

For numbered affiliations see end of article.

Correspondence to

Dr Lesley Ward;

lesley.ward@northumbria.ac.uk

\section{ABSTRACT}

Background The use of yoga as a therapeutic modality is increasing; however, a lack of transparent intervention reporting is restricting the dissemination and implementation of yoga research into clinical and community practice. The aim of this study was to develop a yoga-specific reporting guideline as an extension to existing reporting guidelines for randomised controlled trials, observational studies and case reports.

Methods Recognised international stakeholders in the design and conduct of yoga research were invited to contribute to the electronic Delphi survey. A four-round Delphi was conducted, whereby panellists rated selected items for their importance in the inclusion of yoga reporting guidelines, according to a 5-step Likert scale. A priori consensus for item inclusion was agreement of items as 'Very important' or 'Extremely important' by $\geq 80 \%$ of panellists. Non-consensus items were forwarded to subsequent rounds for re-rating.

Results 53 experts in yoga research from 11 countries, primarily identifying as researchers (50\%), allied health professionals (18.8\%) and yoga professionals (12.5\%), consented to participate in the Delphi. Of these, 48 completed Round 1 (91\%), 43 completed Round 2 (81\%), 39 completed Round 3 (74\%) and 32 completed Round $4(60 \%)$. Panellists reached consensus for inclusion on 21 items, grouped under 10 domains reflective of more generic intervention-based guidelines.

Conclusions The consensus-based 21-item CLARIFY (CheckList stAndardising the Reporting of Interventions For Yoga) checklist provides a minimum reporting template for researchers across a range of methodology designs. Use of these yoga-specific guidelines, in conjunction with the CLARIFY explanation and elaboration guidelines, will standardise the minimum level of detail required for transparent yoga intervention, facilitating the replication, dissemination and implementation of yoga research. Ongoing research will assess the uptake and impact of CLARIFY, to ensure these guidelines retain their relevance to the internationally growing field of yoga research.

\section{INTRODUCTION}

The popularity of yoga as a therapeutic modality has increased dramatically in recent years; with recent surveys indicating $11.8 \%$ and $14.3 \%$ of the adult population in India
Strengths and limitations of this study

A robust, standardised Delphi process was conducted targeting international experts in yoga research.

- Panellists included 53 yoga researchers and yoga therapists from 11 countries across 5 continents.

- Ninety-two per cent of panellists had a minimum of 5 years' experience in yoga research.

- Findings are limited by a higher weighting of North American panellists.

and USA, respectively, practice yoga for health reasons. ${ }^{12}$ The prevalence of yoga use increased linearly from 2002 to 2017, so that it now is one of the most commonly used complementary medicine approaches in the USA. ${ }^{13}$ This increased use is paralleled by an increase in published yoga research, ${ }^{45}$ with promising evidence for the role of yoga in improving symptoms and health-related quality of life across a range of health areas including musculoskeletal, ${ }^{6}$ oncological ${ }^{7}$ and mental health conditions. ${ }^{8}$

Yoga is an umbrella term for various practices, encompassing a range of physical postures, breathing techniques, meditation and lifestyle advice. ${ }^{9}$ Additionally, the content of, and emphasis on, these practices may vary according to the style of yoga being delivered. These elements of variability highlight the importance of adequate reporting in yoga research to enable study replication, summary research, evidence-informed practice and appropriate policy decisions. However, reporting of yoga interventions is often lacking in transparency ${ }^{10}{ }^{11}$ restricting the dissemination and implementation of yoga research in clinical and community practice.

In general, the introduction of several guidelines over the previous decade have been instrumental in improving research conduct and reporting. ${ }^{12}$ Reporting 
guidelines to date have mainly focused on issues specific to certain research methodologies, such as CARE for case reports, ${ }^{13}$ STROBE (Strengthening the Reporting of Observational Studies in Epidemiology) for observational studies $^{14}$ and CONSORT (Consolidated Standards of Reporting Trials) for randomised trials. ${ }^{15}$ While reporting quality in randomised yoga trials has increased following the publication of the revised CONSORT statement, ${ }^{10}$ transparent reporting remains limited in yoga studies. ${ }^{10}$ Specifically, inadequate reporting of intervention content often hinders replication and implementation in clinical practice. $^{11}$

Guideline extensions have been created to address reporting issues specific to interventions such as acupuncture, ${ }^{16}$ exercise $^{17}$ and herbal products. ${ }^{18}$ However, no specific reporting guideline exist for yoga research to date. In response to that need, the aim of this study was to develop a yoga-specific reporting guideline as an extension to existing reporting guidelines for randomised controlled trials, observational studies and case reports.

\section{METHODS}

\section{Study design}

In accordance with published research guidelines, and acknowledging the geographical diversity of potential panellists, the CLARIFY (CheckList stAndardising the Reporting of Interventions For Yoga) guidelines were developed using a consensus-based Delphi approach. Individuals recognised as experts in the field of yoga research were invited to complete several rounds of an electronically delivered survey, rating items for their importance in the reporting of yoga research. Items reaching a priori consensus were then included in the CLARIFY guidelines. Conduct of the survey integrated the key Delphi features of purposive sampling, panellist anonymity and the iterative rating and analytical feedback of survey items, ${ }^{19}{ }^{20}$ with survey parameters reflecting previous Delphi-based guideline development in the research fields of both reporting ${ }^{172}$ and complementary medicine. ${ }^{22} 23$

All facets of the Delphi study, from panellist identification to study termination, were collectively agreed and overseen by a four-member Steering Committee (LW, DN, HC and SM), purposely selected for their international representation, geographical diversity and experience in Delphi methodology and yoga research. Additionally, each survey round was piloted by a four-member team, and amended as necessary before distributing to the main Delphi panellists. Pilot members were purposely selected, as per the steering committee criteria, to assess each survey for clarity of the phrasing of each item, with specific reference to panellists for whom English was not their first language, and for technical robustness of the electronic survey links.

This study was registered with the EQUATOR network. ${ }^{24}$ All participants provided informed consent.

\section{Patient and public involvement}

Patients and/or the public were not involved in the design, or conduct, or reporting, or dissemination plans of this research.

\section{Selection and recruitment of panelists}

Delphi panellists were purposively sampled to ensure a broad geographical and occupational representation of 'experts' in the field of yoga research. ${ }^{19}{ }^{20}$ Eligibility was determined as individuals involved in the conception, design, delivery or reporting of high-quality yoga research, who were computer-literate, and willing to commit to completion of all rounds of the Delphi survey. To optimise non-biased sampling, potential panellists were identified from database searches for peer-reviewed yoga publications, and additional snowball recruitment enabled invitees to recommend other researchers they considered met the eligibility criteria.

In total, 128 international yoga researchers were identified as corresponding publication authors or study leads, of which 114 had available email addresses. These 114 researchers were emailed an invitation pack comprising an invitation letter, participant information sheet and consent form, inviting themselves and team members on the associated yoga publication or study to take part in the Delphi study. Non-responders were sent one follow-up invitation after 2 weeks. Positive responses were received from 53 panellists, who were recruited to the Delphi study (figure 1).

\section{Procedure}

The Delphi survey was conducted over a 12-month period from October 2018 to October 2019. Prior to each round, panellists received an email with a link to access the survey, together with a Personalised Identification Number to be used in place of their name. Panellists were requested to complete the survey within 2 weeks, with reminder emails sent to non-completers at Day 10 and Day 16.

For each round, panellists rated a series of items for their perceived importance in yoga research reporting guidelines, with the goal to improve yoga research dissemination. Panellists were asked to consider their rating in terms of both their own research experience and for future use across a range of study designs including interventions, observational studies and case reports. Items were rated on a 5-point Likert scale: 'Of no importance', 'Of little importance', 'Important', 'Very important' and 'Extremely important'. As per Delphi methodology, each round was analysed on completion, with the results contributing to the content of the subsequent round. ${ }^{19} 20$ Only panellists who completed a survey round were invited to take part in the subsequent round. ${ }^{22} 23$

\section{Survey content}

The Round 1 survey comprised four sections. First, panellists provided consent to participate in the Delphi study (compulsory) and indicated their preference (yes/no) for acknowledgement as a panellist in associated publications. 


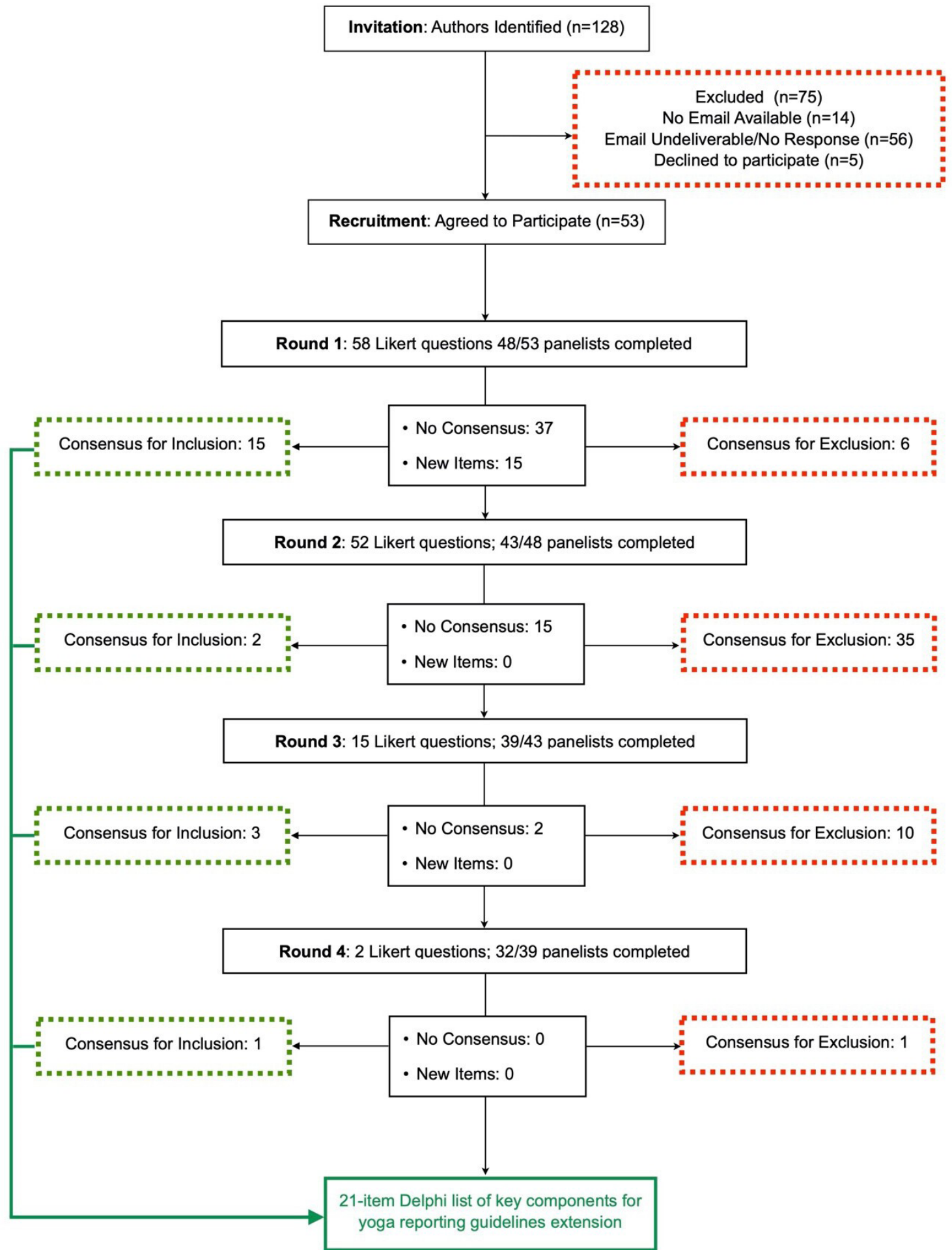

Figure 1 Progression of panellists and items through the four rounds of the Delphi survey.

Second, panellists answered questions relating to demographics, research experience and personal yoga practice, to provide context for the geographical-based and research-based applicability of the CLARIFY guidelines. Third, panellists rated the yoga reporting items on the 5-point Likert scale, with the option of providing free-text comments on each item. Fourth, panellists were provided a space for free-text general comments and suggestions for additional items to include in the subsequent round.
To support consistency across reporting guidelines, the Round 1 items were developed by the Steering Committee in line with the content and grouping of items in intervention reporting guidelines, with specific reference to TIDieR (Template for Intervention Description and Replication) ${ }^{21}$ and CERT (Consensus on Exercise Reporting Template). ${ }^{17}$ Fifty-eight items were produced, grouped into 13 themes of Title (five items); Theory (four items); Materials (three items); Activities (five items); Expertise 
(four items); Delivery (six items); Location (seven items); Dose (five items); Home practice (four items); Adaptations (six items); Protocol modifications (three items); Adherence (one item); and Instructor fidelity (five items).

All subsequent survey rounds comprised three sections: Section 1 summarised the results of the previous round, including a list of items that had reached consensus for inclusion or exclusion in the Delphi guidelines; Section 2 contained items for rating, with the option to comment on them; Section 3 comprised a space for free-text comments.

\section{Analysis, consensus and termination criteria}

Analysis was undertaken at the completion of each survey round and formed the content of the subsequent round's survey. Likert ratings for each item were analysed quantitively and expressed as a percentage for each of the five Likert categories, together with 25, 50 and 75 percentiles (median and IQR). Based on a priori consensus levels, each item was either included in the final Delphi list, excluded from the survey or forwarded to the subsequent round for re-rating. Qualitative, free-text comments were thematically analysed, ${ }^{25}$ and grouped as general comments for addressing in this paper's Discussion, or new items for inclusion in the subsequent round.

A priori consensus levels ensured the CLARIFY guidelines would be based on high levels of panellist agreement. ${ }^{23}$ Accordingly, item consensus for automatic inclusion in the Delphi guidelines was a rating of 'Very important' or 'Extremely important' by $\geq 80 \%$ of panellists, and automatic exclusion from the guidelines was a rating of 'Of no importance' or 'Of little importance' by $\geq 50 \%$ of panellists, or 'Important', 'Very important', or 'Extremely important' by $<75 \%$ of panellists. Nonconsensus items (items not reaching inclusion or exclusion consensus, but rated as 'Important', 'Very important' or 'Extremely important' by $\geq 75 \%$ panellists) were forwarded to the subsequent round for re-rating.

Survey rounds were repeated until no new items were generated from the qualitative data and termination criteria were met for any remaining non-consensus items. Termination criteria were based on inter-round stability of an item according to its median and IQR score. If these scores decreased or remained unchanged between a current and previous round, the item was excluded; if these scores increased then the item was forwarded to the subsequent round for re-rating.

\section{RESULTS}

\section{Demographics of survey completers}

Figure 1 presents the flow of panellists and items through the four rounds of the Delphi survey. Of the 128 yoga researchers identified and contacted by the Steering Committee, 53 consented to participate, of which 48 completed Round 1 (91\%), 43 completed Round 2 (81\%), 39 completed Round 3 (74\%) and 32 completed Round $4(60 \%)$.
The 32 Delphi completers represented 11 countries across North America (59.4\%), Europe (18.8\%), Asia $(6.2 \%)$, Australia $(12.5 \%)$ and South America $(3.1 \%)$ (table 1). Panellists primarily identified as researchers $(50 \%)$, allied health professionals $(18.8 \%)$ and yoga professionals $(12.5 \%)$, with $91.6 \%$ of panellists indicating they practiced yoga. For $62.5 \%$ of panellists, yoga was their primary area of research, with $90.6 \%$ involved in yoga research for at least 5 years. Primary roles within yoga research were as researchers $(93.8 \%)$ and yoga therapists (34.4\%). Demographics of non-completers were similar to completers, with $94 \%$ yoga practitioners, $58.8 \%$ from North America and $52.9 \%$ identifying yoga as their main area of research. Additionally, four of the original six panellists $(67 \%)$ who identified as coming from Asia did not complete the survey beyond Round 1. Demographic data was not provided by the 75 individuals who did not consent or reply to the Delphi invitation.

\section{Item generation and rating}

Over four survey rounds, 21 items reached consensus for inclusion in the CLARIFY guidelines (table 2). The movement of items through the four rounds is outlined below, and data provided in the online supplemental tables.

\section{Round 1}

Forty-eight of the 53 recruited panellists (91\%) provided consent and completed the Round 1 survey. Statistical analysis of the 58 items (online supplemental table 1), based on a priori thresholds, indicated 15 items reached consensus for inclusion in the CLARIFY guidelines, covering eight themes: Theory (one item), Activities (two items), Expertise (one item), Dose (four items), Home Practice (two items), Protocol Modifications (two items), Adherence (one item) and Fidelity (two items). Six items reached consensus for exclusion from the CLARIFY recommendations, under three themes of Title (one item), Delivery (one item) and Location (four items), and the remaining 37 items were forwarded to Round 2 for re-rating.

Forty-eight panellists provided 160 free-text statements, regarding the importance and timeliness of yoga reporting guideline development, the applicability and balance of the current items regarding publication word limits and level of detail required for transparent reporting. Several suggestions referred to items covered by general reporting guidelines, such as assessor-blinding, and were not considered relevant to the specific focus of the CLARIFY guidelines. Based on thematic analysis of this qualitative data, 15 new items were generated for rating in the Round 2 survey, grouped under the themes of Theory (four items), Materials (one item), Activities (one item), Expertise (one item), Delivery (three items), Dose (one item), Home Practice (two items) and Adherence (two items), as denoted with a $\left(^{*}\right)$ symbol in online supplemental table 2. Additionally, the theme Home Practice was added and the theme Delivered As Planned was re-titled Fidelity. 
Table 1 Demographics of the 32 panellists completing all four rounds of the Delphi survey

\begin{tabular}{|c|c|}
\hline & $\mathbf{N}(\%)$ \\
\hline $\mathbf{n}$ & 32 \\
\hline Age (mean (SD)) & $50.41(9.19)$ \\
\hline \multicolumn{2}{|l|}{ Gender (\%) } \\
\hline Female & $21(65.6)$ \\
\hline Male & $11(34.4)$ \\
\hline \multicolumn{2}{|l|}{ Primary occupation (\%) } \\
\hline Academic administrator & $1(3.1)$ \\
\hline $\begin{array}{l}\text { Allied health professional (registered nurse, physical } \\
\text { therapist, occupational therapist, etc) }\end{array}$ & $6(18.8)$ \\
\hline $\begin{array}{l}\text { Integrative health provider (acupuncturist, naturopath, } \\
\text { chiropractor, ayurvedic practitioner) }\end{array}$ & $2(6.2)$ \\
\hline Researcher & $16(50.0)$ \\
\hline Teaching faculty & $3(9.4)$ \\
\hline Yoga professional (instructor, therapist) & $4(12.5)$ \\
\hline \multicolumn{2}{|l|}{ Is yoga the main focus of your research work? (\%) } \\
\hline Yes & $20(62.5)$ \\
\hline \multicolumn{2}{|l|}{ Years involved in yoga research (\%) } \\
\hline $1-5$ & $3(9.4)$ \\
\hline $5-10$ & $16(50.0)$ \\
\hline $10-15$ & $10(31.2)$ \\
\hline 15 or more & $3(9.4)$ \\
\hline \multicolumn{2}{|l|}{ Years of personal yoga practice } \\
\hline Less than 10 years & $4(12.5)$ \\
\hline 10 or more years & $27(84.4)$ \\
\hline \multicolumn{2}{|l|}{$\begin{array}{l}\text { Which yoga practices do you practice? (may select } \\
\text { more than one) (\%) }\end{array}$} \\
\hline Yoga postures (asana) & $29(90.6)$ \\
\hline Breathing exercises (pranayama) & $25(78.1)$ \\
\hline Meditation & $27(84.4)$ \\
\hline Yoga philosophy & 18 (56.2) \\
\hline Yoga nidra & $8(25.0)$ \\
\hline Other & $2(6.2)$ \\
\hline \multicolumn{2}{|l|}{ Continent of origin } \\
\hline Asia & $2(6.2)$ \\
\hline Australia & $4(12.5)$ \\
\hline Europe & $6(18.8)$ \\
\hline North America & $19(59.4)$ \\
\hline South America & $1(3.1)$ \\
\hline \multicolumn{2}{|l|}{ Continent of employment } \\
\hline Asia & $1(3.1)$ \\
\hline Australia & $4(12.5)$ \\
\hline Europe & $3(9.4)$ \\
\hline North America & $23(71.9)$ \\
\hline South America & $1(3.1)$ \\
\hline
\end{tabular}

Which of the following best describes your involvement in yoga research? (may select more than one) (\%)

\begin{tabular}{ll} 
Researcher & $30(93.8)$ \\
Yoga instructor/therapist & $11(34.4)$ \\
Physiotherapist/physical therapist & $2(6.2)$ \\
Statistician & $1(3.1)$ \\
Other & $4(12.5)$ \\
\hline
\end{tabular}

Table 2 Consensus-generated 21-item CLARIFY checklist for the reporting of yoga research

\begin{tabular}{|c|c|c|}
\hline No. & THEME: Subtheme & Item \\
\hline 1 & TITLE & \\
\hline $1 a$ & $\begin{array}{l}\text { Succinctly describe the yoga } \\
\text { intervention. }\end{array}$ & $\begin{array}{l}\text { Include the word 'yoga' in the } \\
\text { publication title. }\end{array}$ \\
\hline 2 & THEORY & \\
\hline $2 a$ & $\begin{array}{l}\text { Describe any rationale, theory or } \\
\text { goal of the elements essential to } \\
\text { the yoga intervention. }\end{array}$ & $\begin{array}{l}\text { Describe why the specific } \\
\text { population was included in the } \\
\text { study. }\end{array}$ \\
\hline 3 & ACTIVITIES & \\
\hline $3 a$ & $\begin{array}{l}\text { Describe the yoga practices } \\
\text { or activities used in the } \\
\text { intervention. }\end{array}$ & $\begin{array}{l}\text { Describe the duration of yoga } \\
\text { practices within yoga session } \\
\text { (eg, } 20 \mathrm{~min} \text { postures, } 10 \mathrm{~min} \\
\text { breathing). }\end{array}$ \\
\hline
\end{tabular}

$3 b$

Describe the type of yoga practices included (eg, postures/ asana, breathing/pranayama meditation, relaxation).

\begin{tabular}{|c|c|c|}
\hline 4 & EXPERTISE & \\
\hline $4 a$ & $\begin{array}{l}\text { Describe the expertise, } \\
\text { background and training of } \\
\text { those providing the yoga } \\
\text { intervention. }\end{array}$ & $\begin{array}{l}\text { Describe the qualifications of the } \\
\text { yoga instructor(s). }\end{array}$ \\
\hline 5 & DELIVERY & \\
\hline $5 a$ & $\begin{array}{l}\text { Describe how the yoga } \\
\text { intervention was delivered (eg, } \\
\text { class, video/audio) and whether } \\
\text { it was provided individually or in } \\
\text { a group. }\end{array}$ & $\begin{array}{l}\text { Describe the teaching approach } \\
\text { including: visual demonstration, } \\
\text { verbal guidance and/or hands on } \\
\text { assistance. }\end{array}$ \\
\hline
\end{tabular}

$\begin{array}{cl}6 & \text { DOSE } \\ 6 \mathbf{a} & \begin{array}{l}\text { Describe the number of } \\ \text { times the yoga intervention } \\ \text { was delivered and over what } \\ \text { period, including the number } \\ \text { of sessions, their schedule and } \\ \text { their duration and intensity. }\end{array}\end{array}$

$6 c$

Describe the duration of each yoga session (in minutes).

Describe the duration of the yoga intervention (eg, over 8 weeks).

Describe the frequency of yoga sessions (eg, two times per week).

$6 d$

Describe the number of yoga sessions.

\begin{tabular}{|c|c|c|}
\hline 7 & HOME PRACTICE & \\
\hline $7 a$ & \multirow[t]{3}{*}{$\begin{array}{l}\text { Describe aspects of home } \\
\text { practice if any. }\end{array}$} & $\begin{array}{l}\text { Describe the duration and } \\
\text { frequency of home practice (if } \\
\text { any). }\end{array}$ \\
\hline $7 b$ & & $\begin{array}{l}\text { Report whether yoga was } \\
\text { available to participants during } \\
\text { the follow-up period (if relevant), } \\
\text { and list any recommendations } \\
\text { made for home practice dose. }\end{array}$ \\
\hline 7c & & $\begin{array}{l}\text { Describe if and how adherence } \\
\text { to home practice was measured. }\end{array}$ \\
\hline 8 & \multicolumn{2}{|l|}{ PROTOCOL CHANGES } \\
\hline $8 a$ & \multirow{2}{*}{$\begin{array}{l}\text { If the yoga intervention was } \\
\text { modified during the course of } \\
\text { the study in ways not described } \\
\text { in the protocol, please describe } \\
\text { the changes. }\end{array}$} & $\begin{array}{l}\text { Describe any changes to the } \\
\text { yoga protocol during the study. }\end{array}$ \\
\hline $8 b$ & & $\begin{array}{l}\text { Describe the rationale for } \\
\text { changes to the yoga protocol } \\
\text { during the study. }\end{array}$ \\
\hline 9 & \multicolumn{2}{|l|}{ PARTICIPANT ADHERENCE } \\
\hline $9 a$ & \multirow{2}{*}{$\begin{array}{l}\text { If adherence to the yoga } \\
\text { intervention was assessed, } \\
\text { describe how and by whom } \\
\text { and what, if any strategies were } \\
\text { used to maintain or improve } \\
\text { adherence. }\end{array}$} & $\begin{array}{l}\text { Describe if and how class/ } \\
\text { session attendance was } \\
\text { measured. }\end{array}$ \\
\hline $9 b$ & & $\begin{array}{l}\text { Describe any strategies used to } \\
\text { promote practice adherence. }\end{array}$ \\
\hline
\end{tabular}




\begin{tabular}{lll}
\hline Table 2 & Continued & \\
\hline No. & THEME: Subtheme & Item \\
\hline 10 & INSTRUCTOR FIDELITY & \\
\hline 10a & $\begin{array}{l}\text { Describe the extent to which the } \\
\text { yoga intervention was delivered } \\
\text { as planned. }\end{array}$ & $\begin{array}{l}\text { Describe the assessment of } \\
\text { protocol fidelity. }\end{array}$ \\
10b & $\begin{array}{l}\text { Describe the reasons for } \\
\text { deviation from study plan. } \\
\text { Describe any differences } \\
\text { between proposed programme } \\
\text { and actual programme delivery. } \\
\text { Describe when protocol was } \\
\text { modified. }\end{array}$ \\
\hline 10d & & \\
\hline
\end{tabular}

Round 2

Forty-three panellists completed the 52-item Round 2 survey (online supplemental table 2). Two of the 52 items (one each from Title and Protocol Fidelity theme) reached consensus for inclusion, and 34 items across all themes except Adherence reached consensus for exclusion. The remaining 16 items met a priori criteria for forwarding to Round 3 for re-rating.

Twenty-nine panellists provided 112 free-text comments. These primarily sought clarification between participant adherence to the intervention (relating to items under the theme of Attendance) and interventionist adherence to the protocol (relating to items under Fidelity). Additionally, some panellists questioned the use of the term describe in the wording of the items, suggesting it would require authors to provide too much detail when writing up their publications. Panellists also highlighted current tensions in yoga research between strict adherence to a study protocol and individualised tailoring to study participants, with comments regarding the pragmatics of detailing participant-centred variations inherent to the delivery of a yoga class. Based on thematic analysis of the qualitative data no new items were added; however, two of the forwarded items under the theme 'Home practice' were found to have similar wording and were combined into one item for Round 3. Additionally, 4 of the 15 forwarded items and two of the forwarded theme labels were re-worded for clarity (as indicated with a $(*)$ symbol in online supplemental table 3).

\section{Round 3}

Thirty-nine panellists completed the 15-item Round 3 survey. Three items reached consensus for inclusion within the themes of Home Practice, Adherence and Instructor Fidelity. Of the remaining 12 items, 2 items met forwarding criteria based on inter-round stability (one each in the themes of Adaptations and Delivery), and 10 items reached consensus for exclusion in the themes of Theory, Activities, Expertise, Delivery, Home Practice and Participant Adherence.

Twenty panellists provided 65 free-text comments, again mostly related to disparate viewpoints regarding the level of detail required in intervention reporting. In contrast to Round 2 comments regarding over-detailed reporting and the accompanying exclusion of many related items,
Round 3 comments indicated other panellists disagreed and considered too much detail had now been removed. Following thematic analysis, no new items were generated from the qualitative data, no items were re-worded and two themes were renamed for clarification: Adherence was renamed Participant Adherence, and Protocol Fidelity was renamed Instructor Fidelity.

\section{Round 4}

Thirty-two panellists responded to the final two-item survey round. One item within the Delivery theme reached consensus for inclusion, and the item under the Adaptations theme was excluded based on interround stability criteria. Nineteen panellists provided 32 comments, again related to disparate views regarded the optimal level of detail for yoga intervention reporting. Thematic analysis generated no new items, and termination criteria for the Delphi survey was fulfilled.

\section{DISCUSSION}

This study addressed the impact of suboptimal intervention reporting on the dissemination and implementation of yoga research, by developing yoga-specific reporting guidelines applicable across a range of research designs. Thirty-two identified experts in yoga research from 11 countries across 5 continents completed four rounds of a Delphi survey, quantitively rating and qualitatively refining survey items. The resultant consensus-based CLARIFY guidelines provide a 21-item, yoga-specific list for minimum reporting of yoga standards, grouped under 10 domains reflective of more generic intervention-based guidelines. ${ }^{17} 21$ The demographic diversity of Delphi panellists supports both the international applicability of the CLARIFY guidelines, and their use for both the providers (researchers) and end users (yoga therapists and yoga teachers) of yoga research.

The breadth and depth of reporting guidelines presents as a contentious issue, and within this study there was noted disparity among Delphi panellists regarding the amount of detail considered necessary for yoga intervention reporting. Comments indicated that most panellists favoured minimal detail, leading to the exclusion of several items during Round 2 of the survey; however, this was countered by opposing views in Round 3, whereby other panellists considered too much detail had been removed. With regard to this, we emphasise that the 21-item CLARIFY checklist, in line with other reporting guidelines,${ }^{27}$ is a minimum, as opposed to an exhaustive, guideline. Specially, CLARIFY provides a consensus-based reporting level that enables independent replication and implementation of a yoga intervention in clinical practice. However, as the necessary depth of information will vary between interventions and study designs, individual researchers are encouraged to report additional detail as perceived necessary. To assist with this, we have provided a table of excluded items rated as 'Very important + Extremely important' by more than $50 \%$ of panellists, to 
indicate items that received majority, but not consensus ratings (online supplemental appendix 1). Given that this data may be presented in various forms, including tables, figures or supplementary text, journal word limits will preclude adequate reporting in only rare exceptions.

The CLARIFY reporting items with the highest consensus for inclusion were related to five themes of dose, home practice, protocol modification, participant adherence to the yoga intervention and instructor fidelity to the intervention. Of note, panellists reached immediate consensus for most of these items in Round 1 of the survey. Dose is generally considered as key information in study interventions. ${ }^{21} 2328$ While dose-response relationships within yoga research have been primarily limited to individual yoga trials, ${ }^{29}{ }^{30}$ a recent meta-analysis of trial data suggests a dose-effectiveness relationship across yoga interventions. ${ }^{31}$ As clinical guidelines increasingly recommend yoga for therapeutic health management, for example, ${ }^{32} 33$ improved reporting of dosage parameters may further elucidate this relationship and provide information to medical professionals regarding the prescriptive use of yoga for clinical benefit. Intervention dosage may include individual home practice, an area inadequately reported in yoga research. ${ }^{11}$ The CLARIFY panellists have addressed this gap with the inclusion of three items related to the content and dosage of home practice. Reporting of these home practice items will additionally improve transparency, and thus independent replication, of yoga interventions, fulfilling a basic requirement for making generally applicable treatment recommendations. ${ }^{34}$

Further supporting trial replication and implementation are the CLARIFY items relating to protocol modification and deviations. Research should not only report what was planned a priori, but also what was finally delivered, to provide a broader understanding of the actual conduct of a study. ${ }^{35}$ Protocol deviations can occur on the level of the investigator or therapist (classified here as Fidelity) or on the level of the participant (classified here as Adherence). Highlighting these pragmatic issues will aid both the design of future yoga interventions and the translation and dissemination of yoga research into the health professional and lay communities.

Panellists were also in agreement on several items they considered as unimportant for yoga reporting guidelines, reaching quick consensus to exclude these items within the first two rounds of the survey. These items predominantly related to pragmatic aspects of the design and delivery of a yoga intervention, specifically the location (environment and accessibility), timing and provision of course materials for the yoga classes. Information regarding the lineage of the yoga intervention and yoga teachers were also excluded.

An interesting aspect of Delphi surveys is the differing and immutable viewpoints of the panellists, as indicated by inter-round stability of the items rating scores. For this Delphi survey, differing viewpoints among panellists resulted in several items being excluded from the final CLARIFY reporting guidelines due to nonconsensus. As above, some of these items related to pragmatic aspects of design and delivery. Additionally, several non-consensus items addressed the depth of reporting detail required for yoga interventions. While broader aspects of yoga content, such as describing the type of yoga practices were included in the CLARIFY guidelines, further detail such as the pace, sequencing and modification of the yoga practices did not reach consensus.

To assess any potential demographic bias in the exclusion of items, an exploratory analysis was conducted to determine whether panellist responses differed by Country of Origin (USA vs non-USA), Occupation (researcher vs non-researcher) and Research Experience (1-10 vs $>10$ years) for items that were excluded from, or did not reach consensus for, the CLARIFY guidelines. Country of origin differences were noted regarding the level of detail of the yoga intervention. While there is likely greater diversity of perspective within this category delineation of USA versus non-USA panellist, there are interesting trends worth considering in future research and reporting priorities. Yoga researchers of non-USA origin tended to place greater emphasis on the level of detail required for reporting the content of the yoga intervention, such as naming, duration and repetitions of individual postures and breathing techniques. Conversely, US panellists tended to place greater importance on conceptual matters, such as the foundational theoretical model for the intervention and the rationale for protocol changes.

Researchers and non-researchers opinions differed in two main aspects of reporting yoga interventions. Panellists identifying as non-researchers were most likely to be yoga interventionists, consultants and subject matter experts whose primary expertise was in the delivery of yoga rather than the scientific investigation of it. They tended to rate more highly the rationale for choosing a specific yoga approach, and the detailed description of the pace the yoga practices were delivered with. Researchers, on the other hand, were more likely than non-researchers to place importance on items delivered external to instructor-supervised classes, including use of materials for home practice, yoga availability during follow-up and recommendations for home practice.

Panellists' research experience appeared to have little influence on their responses. However, panellists with less (1-10 years) research experience placed more importance on reporting potential mechanisms of action for the specific yoga approach and detailing how session adherence was documented than those with more ( $>10$ years) research experience. Based on free-text comments, it appeared the more experienced researchers were keenly aware of word limitations for manuscript submissions and therefore more likely to rate some items as less critical than their less experienced counterparts. 


\section{Limitations}

A primary limitation of the development of Delphibased guidelines is the breadth of representation of experts in the relevant field. To limit any demographic bias, participants were primarily identified from independently published yoga research and trial registry databases, supplemented with snowball recruitment. While geographical representation was gained across five continents, there was a noted North American bias in panellists' country of employment. Additionally, participant attrition increased across the final two rounds of the survey. Analysis indicated similar demographics between completers and non-completers; however, it was unclear if attrition was related to survey fatigue or to disagreement with results from the previous round.

These study limitations are not unexpected and will be addressed as part of the ongoing development and refinement of the 21-item CLARIFY guidelines. An explanation and elaboration paper $^{36}$ has been developed to assist researchers in the pragmatic use of the CLARIFY guidelines, and an analysis of changes in reporting of yoga interventions will be undertaken following an adequate time period, to assess the impact of the guidelines and refine based on user feedback. ${ }^{16}$

\section{Conclusion}

The current Delphi study engaged 32 identified experts to develop guidelines addressing the transparency of intervention reporting in yoga research. The resultant consensus-based, 21-item CLARIFY checklist provides a reporting template for researchers across a range of methodology designs. We recommend the use of these guidelines to standardise the minimum level of detail to provide in future yoga research. We anticipate use of the CLARIFY checklist, in conjunction with the CLARIFY explanation and elaboration guidelines, will facilitate the replication, dissemination and implementation of yoga research. Ongoing research will expand our panellist groups to include integrative medicine practitioners and patient representatives to assess the uptake and impact of CLARIFY. This broader representation will ensure these guidelines retain their relevance to all stakeholders involved in the internationally growing field of yoga research.

\section{Author affiliations}

${ }^{1}$ Department of Sport, Exercise and Rehabilitation, Northumbria University, Newcastle upon Tyne, UK

${ }^{2}$ Australian Research Centre in Complementary and Integrative Medicine, Faculty of Health, University of Technology Sydney, Sydney, New South Wales, Australia

${ }^{3}$ Department of Integrative Health Research, Maryland University of Integrative Health, Laurel, Maryland, USA

${ }^{4}$ Department of Internal and Integrative Medicine, Evang, Kliniken Essen-Mitte, Faculty of Medicine, University of Duisburg-Essen, Essen, Germany

Acknowledgements We gratefully acknowledge the following people for their input into the development of the CLARIFY guidelines. For input into the identification of potential panellists: David Riley, Laura Schmalzl, Rani Elwy and Erik Grossl. Panellists who piloted all rounds of the Delphi survey: Eda Tonga, Romy Lauche and two panellists who wish to remain anonymous. The 32 panellists who contributed to all rounds of the Delphi survey: Timothy Avery, Kylie Barr, Susan Bartlett, Peter Bayley, Christiane Brems, Yvonne Colgrove, Michael Demanincor, Jeffery Dusek, Steriani Elavsky, Erik Groessl, Marshall Hagins, Zoe Hewett, Kim Innes, Sara Lazar, Casey Mace-Firebaugh, Megan Maiya, Roseny Flávia Martins, Kimberly Middleton, Jani Mikkonen, Charlene Muhammad, James Newham, Barry Oken, JuYoung Park, Abbas Rakhshani, Arlene Schmid, Karen Sherman, Vinoy Singh, Chris Streeter, Marlysa Sullivan, Anne Tiedemann, Laura Todd and Marieke Van Puymbroeck. Additionally, we would like to acknowledge and thank those panellists who participated in rounds 1 through 3 of the survey. For assistance with early trial administration, Melissa Smith.

Contributors LW acted as guarantor of the study. SM and LW were responsible for conception and design of the work, interpretation of the data, participated in acquisition and analysis and drafted the manuscript. DN and $\mathrm{HC}$ participated in the conception and design of the work, and interpretation of the data, were responsible for acquisition and analysis of the data, and critically revised the manuscript. All authors gave final approval of the version to be published, and agree to be accountable for all aspects of the work.

Funding Research reported in this publication was partially supported by the National Center for Complementary and Integrative Health of the National Institutes of Health under award number R24 AT001293.

Competing interests None declared.

Patient consent for publication Not applicable.

Ethics approval This study involves human participants and was approved by Institutional Review Board at the Maryland University of Integrative Health (07. M00.06.18.2). Participants gave informed consent to participate in the study before taking part.

Provenance and peer review Not commissioned; externally peer reviewed.

Data availability statement Data are available upon reasonable request. Data is supplied in the supplementary files.

Supplemental material This content has been supplied by the author(s). It has not been vetted by BMJ Publishing Group Limited (BMJ) and may not have been peer-reviewed. Any opinions or recommendations discussed are solely those of the author(s) and are not endorsed by BMJ. BMJ disclaims all liability and responsibility arising from any reliance placed on the content. Where the content includes any translated material, BMJ does not warrant the accuracy and reliability of the translations (including but not limited to local regulations, clinical guidelines, terminology, drug names and drug dosages), and is not responsible for any error and/or omissions arising from translation and adaptation or otherwise.

Open access This is an open access article distributed in accordance with the Creative Commons Attribution Non Commercial (CC BY-NC 4.0) license, which permits others to distribute, remix, adapt, build upon this work non-commercially, and license their derivative works on different terms, provided the original work is properly cited, appropriate credit is given, any changes made indicated, and the use is non-commercial. See: http://creativecommons.org/licenses/by-nc/4.0/.

\section{ORCID iDs}

Holger Cramer http://orcid.org/0000-0002-3640-8046

Steffany Moonaz http://orcid.org/0000-0003-3143-1631

\section{REFERENCES}

1 Clarke TC, Barnes PM, Black LI. Use of yoga, meditation, and chiropractors among US adults aged 18 and over. NCHS Data Brief 2018:1-8.

2 Mishra AS, SK R, HS V, et al. Knowledge, attitude, and practice of yoga in rural and urban India, KAPY 2017: A nationwide cluster sample survey. Medicines 2020;7:8.

3 Clarke TC, Black LI, Stussman BJ, et al. Trends in the use of complementary health approaches among adults: United States, 2002-2012. Natl Health Stat Report 2015;79:p. 1-16.

4 Cramer H, Lauche R, Dobos G. Characteristics of randomized controlled trials of yoga: a bibliometric analysis. BMC Complement Altern Med 2014;14:328.

5 Jeter PE, Slutsky J, Singh N, et al. Yoga as a therapeutic intervention: a bibliometric analysis of published research studies from 1967 to 2013. J Altern Complement Med 2015;21): :586-92.

6 Wieland LS, Skoetz N, Pilkington K, et al. Yoga treatment for chronic non-specific low back pain. Cochrane Database Syst Rev 2017;1:Cd010671. 
7 Pan Y, Yang K, Wang Y. Could yoga practice improve treatmentrelated side effects and quality of life for women with breast cancer? A systematic review and meta-analysis. Asia-Pacific Journal of Clinical Oncology 2017;13:e79-95.

8 Cramer H, Lauche R, Anheyer D, et al. Yoga for anxiety: a systematic review and meta-analysis of randomized controlled trials. Depress Anxiety 2018;35): :830-43.

9 Feuerstein G. The Yoga Tradition. Prescott: Hohm Press, 1998.

10 Cramer $\mathrm{H}$, Langhorst J, Dobos G, et al. Associated factors and consequences of risk of bias in randomized controlled trials of yoga: a systematic review. PLoS One 2015;10:e0144125.

11 Ward L, Stebbings S, Cherkin D, et al. Components and reporting of yoga interventions for musculoskeletal conditions: a systematic review of randomised controlled trials. Complement Ther Med 2014;22): :909-19.

12 Mercieca-Bebber R, Rouette J, Calvert M, et al. Preliminary evidence on the uptake, use and benefits of the CONSORT-PRO extension. Qual Life Res 2017;26:1427-37.

13 Gagnier JJ, Kienle G, Altman DG, et al. The care guidelines: consensus-based clinical case reporting Guideline development. Headache 2013:53:1541-7.

14 von Elm E, Altman DG, Egger M, et al. The strengthening the reporting of observational studies in epidemiology (STROBE) statement: guidelines for reporting observational studies. Ann Intern Med 2007;147:573-7.

15 Schulz KF, Altman DG, Moher D, et al. Consort 2010 statement: updated guidelines for reporting parallel group randomized trials. Ann Intern Med 2010;152:726-32.

16 MacPherson H, Altman DG, Hammerschlag R, et al. Revised standards for reporting interventions in clinical trials of acupuncture (stricta): extending the CONSORT statement. PLoS Med 2010;7:e1000261.

17 Slade SC, Dionne CE, Underwood M, et al. Consensus on exercise reporting template (CERT): modified Delphi study. Phys Ther 2016;96:1514-24.

18 Gagnier JJ, Boon H, Rochon P, et al. Reporting randomized, controlled trials of herbal interventions: an elaborated consort statement. Ann Intern Med 2006;144:364-7.

19 Hasson F, Keeney S, McKenna H. Research guidelines for the Delph survey technique. J Adv Nurs 2000;32:1008-15.

20 Keeney S, Hasson F, McKenna H. Consulting the oracle: ten lessons from using the Delphi technique in nursing research. J Adv Nurs 2006:53:205-12.

21 Hoffmann TC, Glasziou PP, Boutron I, et al. Better reporting of interventions: template for intervention description and replication (TIDieR) checklist and guide. BMJ 2014;348:g1687.
22 Goldenberg JZ, Ward L, Day A, et al. Naturopathic approaches to irritable bowel syndrome-a Delphi study. J Altern Complement Med 2019;25:227-33.

23 Ward L, Stebbings S, Sherman KJ, et al. Establishing key components of yoga interventions for musculoskeletal conditions: a Delphi survey. BMC Complement Altern Med 2014;14:1-12.

24 Equator Network. Reporting guidelines under development for clinical trials, 2019. Available: https://www.equator-network.org/ library/reporting-guidelines-under-development/reporting-guidelinesunder-development-for-clinical-trials/\#YOGA

25 Braun V, Clarke V. Using thematic analysis in psychology. Qual Res Psychol 2006;3:77-101.

26 Whiting P, Rutjes AWS, Reitsma JB, et al. The development of QUADAS: a tool for the quality assessment of studies of diagnostic accuracy included in systematic reviews. BMC Med Res Methodol 2003;3:25.

27 Equator Network. What is a reporting guideline? 2020. Available: https://www.equator-network.org/about-us/what-is-a-reportingguideline/ [Accessed 12 Apr 2021].

28 Tate DF, Lytle LA, Sherwood NE, et al. Deconstructing interventions: approaches to studying behavior change techniques across obesity interventions. Transl Behav Med 2016;6:236-43.

29 Michalsen A, Jeitler M, Brunnhuber S, et al. lyengar yoga for distressed women: a 3-armed randomized controlled trial. Evid Based Complement Alternat Med 2012;2012:1-9.

30 Saper RB, Boah AR, Keosaian J, et al. Comparing Onceversus twice-weekly yoga classes for chronic low back pain in predominantly low income minorities: a randomized dosing trial. Evid Based Complement Alternat Med 2013;2013:1-13

31 Brinsley J, Schuch F, Lederman O, et al. Effects of yoga on depressive symptoms in people with mental disorders: a systematic review and meta-analysis. Br J Sports Med 2021;55:992-1000.

32 National Institute for Health and Care Excellence. Multiple sclerosis in adults: management. clinical guideline, 2020. Available: https:// www.nice.org.uk/guidance/cg186

33 Qaseem A, Wilt TJ, McLean RM, et al. Noninvasive treatments for acute, subacute, and chronic low back pain: a clinical practice guideline from the American College of Physicians. Ann Intern Med 2017; $166: 514-30$

34 Schmidt S. Shall we really do it again? the powerful concept of replication is neglected in the social sciences. Review of General Psychology 2009;13:90-100.

35 Ghooi RB, Bhosale N, Wadhwani R, et al. Assessment and classification of protocol deviations. Perspect Clin Res 2016;7:132-6.

36 Moonaz Set al. Explanation and elaboration of the clarify (checklist stAndardising the reporting of interventions for yoga) guideline. BMJ Open 2021;11:e045812. 